\title{
Automated Nomenclature Labeling of the Bronchial Tree in 3D-CT Lung Images
}

\author{
Hiroko Kitaoka ${ }^{1,6}$, Yongsup Park ${ }^{2}$, Juerg Tschirren ${ }^{3}$, Joseph Reinhardt ${ }^{4}$, \\ Milan Sonka ${ }^{3}$, Goeffrey McLennan ${ }^{5}$, and Eric A. Hoffman ${ }^{1,4}$ \\ ${ }^{1}$ Division of Physiologic Imaging, Dept. of Radiology, College of Medicine, \\ University of Iowa, 200 Hawkins Drive, Iowa City, Iowa 52242, USA \\ \{hiroko-kitaoka, eric-hoffman\} @uiowa.edu \\ ${ }^{2}$ Dept. of Informatics and Mathematical Science, Graduate School of Engineering Science, \\ Osaka University, 2-2 Yamadaoka, Suita, Osaka 363-0871, Japan \\ yspark@image.med.osaka-u.ac.jp \\ ${ }^{3}$ Dept. of Electrical and Computer Engineering, \\ College of Engineering, University of Iowa, 1402 SC, Iowa City, Iowa 52242, USA \\ \{juerg-tschirren, milan-sonka\} @uiowa . edu \\ ${ }^{4}$ Dept. of Biomedical Engineering, College of Engineering, University of Iowa, \\ 1402 SC, Iowa City, Iowa 52242, USA \\ joe-reinhardt@uiowa.edu \\ ${ }^{5}$ Dept. of Internal Medicine, College of Medicine, the University of Iowa, \\ 200 Hawkins Drive, Iowa City, Iowa 52242, USA \\ geoffrey-mclennan@uiowa. edu \\ ${ }^{6}$ Biomedical Physics Laboratory, Brussels Free University, \\ Campus Erasme cp 613/3, 808 Route de Lennik, 1070 Brussels, Belgium
}

\begin{abstract}
A nomenclature labeling algorithm for the human bronchial tree down to sub-lobar segments is proposed, as a means of inter and intra subject comparisons for the evaluation of lung structure and function. The algorithm is a weighted maximum clique search of an association graph between a reference tree and an object tree. The adjacency between nodes in the association graph is defined so as to reflect the consistency between the bronchial name in the reference tree and the node connectivity in the object tree. Nodes in the association graph are weighted according to the similarity between two tree nodes in the respective trees. This algorithm is robust to various branching patterns and false branches that arise during segmentation processing. Experiments have been performed for nine airway trees extracted automatically from clinical 3D-CT data, where approximately 250 branches were contained. Of these, $95 \%$ were accurately named.
\end{abstract}

\section{Introduction}

Isotropic volume data acquisition for medical imaging is now rapidly spreading in clinical use due to the technological progress in multi-detector CT scanners. 3D image processing techniques have enabled precise structural analysis of living organs. Anatomical nomenclature is an important step in sharing a common understanding of organ structure. Inter-individual and intra-individual comparisons are meaningful only when accurate nomenclatures are applied to the structures. Accuracy of nomenclature is also critical for diagnosis and surgical planning. However, anatomical knowledge used for establishing the nomenclature of biological structure is challenging when seeking to construct robust computational algorithms, because of the nature of bio- 
logic complexity and diversity. Discrepancy of anatomical nomenclature even between experts is not uncommon.

The human airway tree is a typical example of the difficulty of nomenclature and labeling because of its hierarchical properties and the considerable variations of branching pattern. Mori et al reported a knowledge-based labeling method of the bronchial branches and applied it to seven cases of CT images with a slice thickness of 2 or $3 \mathrm{~mm} \mathrm{[1].} \mathrm{In} \mathrm{their} \mathrm{experiment,} \mathrm{the} \mathrm{number} \mathrm{of} \mathrm{extracted} \mathrm{branches} \mathrm{for} \mathrm{each} \mathrm{sub-}$ ject was about thirty, and none of extracted trees from the seven cases had all segmental bronchi. By the use of modern multi detector CT scanners, more than a hundred bronchial branches can be extracted. The increase in the number of branches identified increases the complexity of establishing a robust labeling scheme.

In this paper, we first explain how the bronchial nomenclature is constructed in terms of graph theory, and introduce an algorithm based on a weighted maximum clique search of an association graph between a reference tree and an object tree. We demonstrate its performance by volumetric human lung CT data sets. We believe the proposed algorithm will be applicable to tree systems not only in the lung but also in other organs and across species.

\section{Principles of Bronchial Nomenclature}

\subsection{General Aspects of Bronchial Nomenclature}

The human airway tree begins from the trachea and repeatedly branches into smaller and smaller bronchi, ending in the terminal bronchioles, whose diameter is about 0.5 $\mathrm{mm}$. The total number of the airway branches is over 50,000 in the normal adult human [2], and the bronchial nomenclatures are defined to 74 proximal branches down to sub-segmental bronchi [3], [4 ], [5 ]. Currently, the most clinically important nomenclatures include 32 branches down to segmental bronchi. Peripheral bronchi that lie downstream from a segmental bronchus are usually named using the nomenclature of the parent segmental bronchi. The bronchial nomenclature is assigned according to the region of the lung to which a bronchus supplies air. There is a clear definition for the spatial division of the lung as shown in Figure 1. Classes of lobe, segment, and subsegment construct a hierarchic structure, and a set of all members in the same class is equal to the whole lung without overlapping. There is an exact one-to-one correspondence between a branch and the lung region supplied air by the branch, because there are no loops in the airway tree. Therefore, the bronchial nomenclatures are based upon the regional nomenclatures: lobar bronchus, segmental bronchus, and so on.

The most common way to mathematically describe the airway tree is by a graph representation using a rooted tree. However, for the purpose of bronchial nomenclature, a tree representation can lead to confusion, because the hierarchy of the rooted tree does not correspond to the nomenclature hierarchy. Figure 2 shows a standard branching pattern of the human bronchial tree [3], where thick lines indicate bronchi having anatomical nomenclatures. In this branching pattern, levels of segmental bronchi across from $3^{\text {rd }}$ to 7 th. Furthermore, as shown in Figure 3, there are differences in branching patterns even across normal subjects. It is obvious that the same nomenclature does not mean the same level in the tree representations of different branching patterns. 


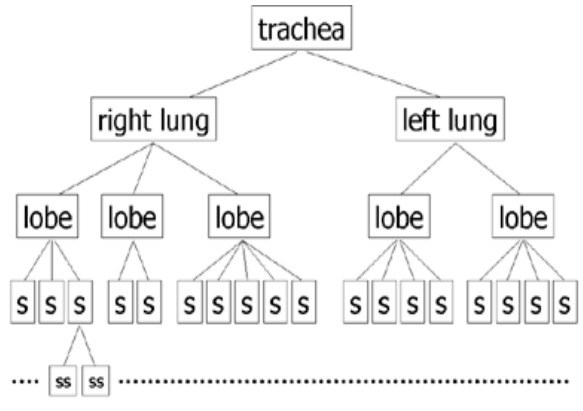

Fig. 1. Hierarchy of space division of the lung. s: lung segment. ss: sub-segment

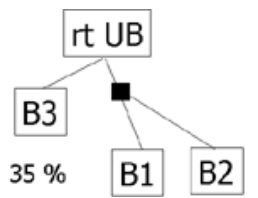

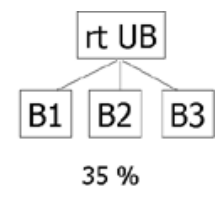

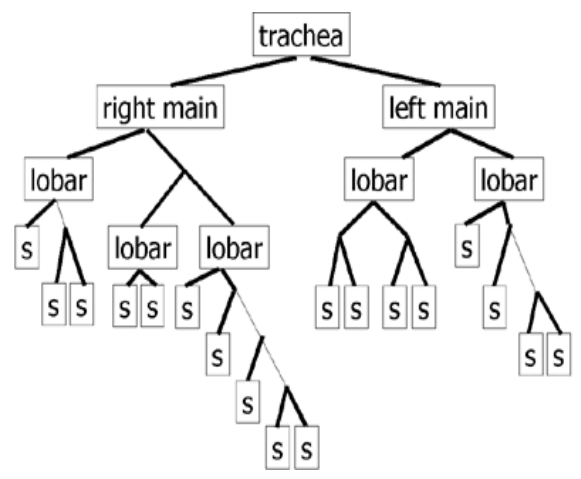

Fig. 2. A typical example of the human bronchial tree. s: segmental bronchus

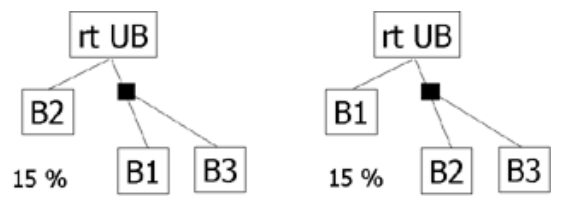

Fig. 3. Branching patterns of segmental bronchi arising from the right upper lobar bronchus (UB). Frequencies for respective branching patterns are according to [3]

Since there are only five lobar bronchi and there is little variation in the branching pattern, nomenclatures for lobar bronchi are not difficult. On the other hand, determining nomenclatures for segmental bronchi are much more difficult because of a large variety of branching patterns.

\subsection{Nomenclature of the Segmental Bronchus}

There are ten lung segments in the right lung and eight segments in the left lung. The names of lung segments describe their locations within the lung. For example, there are apical, lateral, and anterior segments of the right upper lobe. For simplicity, numbers from 1 to 10 are used for distinguishing locations. Both the right and left lower lobes sometimes have accessory segments called sub-superior segments, which are often located below the superior segmental bronchus (B6). They are usually expressed as the symbol of asterisk (*) instead of number [3], [4], [5]. Since branchpoints in the bronchial tree have only one upward branch, it is reasonable to assign bronchial nomenclatures to branchpoints, as shown in Figure 4.

As shown in Figure 4, each segmental bronchus is located neither upstream nor downstream from other segmental bronchi, since their supplying regions are independent of each other. In addition, the segmental bronchi are always located distal to their parent lobar bronchi regardless of the branching order, because each lobe is comprised of its member segments. These two relationships appear trivial but are very important for clarifying the node connectivity in a rooted tree in terms of graph theory. Meanwhile, intermediate branches between lobar and segmental bronchi have no 
anatomical names because of their ambiguous relationships. These relationships do not change even if a tree contains false branches or missing true branches due to image processing steps including segmentation and skeletonization. Errors occurring in the segmentation and skeletonization steps of image processing algorithms serve as a primary source of difficulty when seeking to automatically label the bronchial tree.

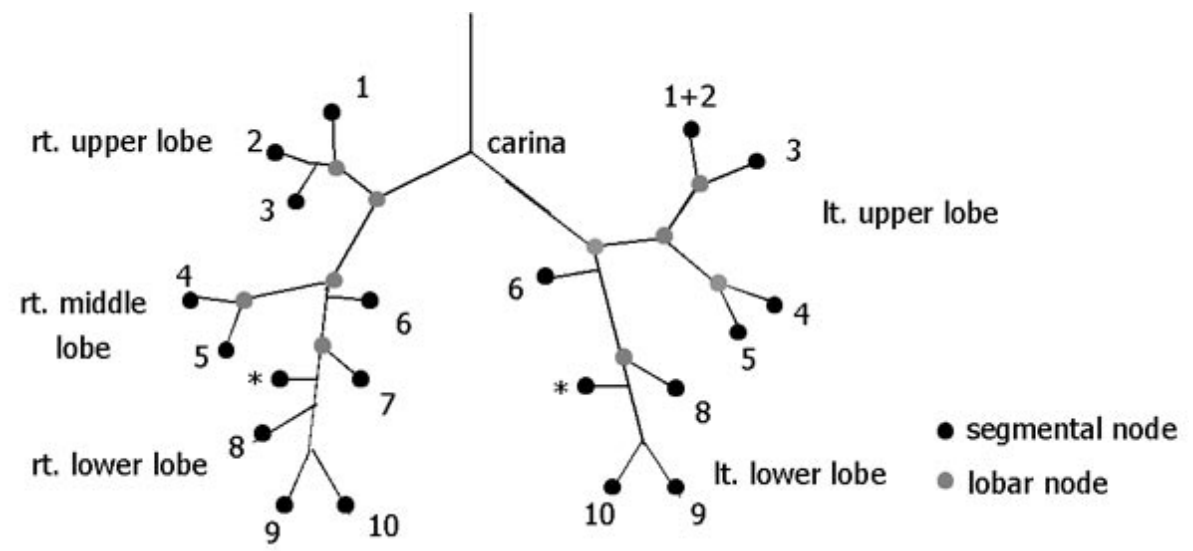

Fig. 4. Scheme of the bronchial nomenclatures. Each segmental node is connected upward to the segmental bronchi. Some of lobar nodes presented here are different from the traditional definitions. See the text in 3.3

There is one more important characteristic of the bronchial nomenclature that can provide node attributes in the airway tree. Each lung segment is supplied air by its corresponding segmental bronchus, and all branches within the segment are ancestors of the segmental bronchus. Therefore, the position and the direction of a segmental bronchus correspond to the position and the central axis of its associated lung segment. The segmental bronchial nomenclature is defined according to this correspondence, regardless of its branching order.

\section{Bronchial Nomenclature Algorithm}

Automated bronchial nomenclature and labeling can be viewed as a tree matching problem between an object tree and a standard airway tree. The nomenclature labeling is then applied to give the same name to a node in the object tree as that of its corresponding node in the reference tree. The algorithm is based on a weighted tree matching method proposed by Pelillo et al.[6]. Their method seeks the maximum weight clique in a tree association graph (TAG), equivalent to the maximum similarity subtree isomorphism between two trees. We modify the definition of adjacency of TAG nodes and construct a similarity measure between a reference tree and an object tree according to the property of the bronchial nomenclature explained in the previous section. Before explaining our algorithm, Pelillo's original method is briefly described. 


\subsection{Weighted Tree Association Graph by Pelillo et al.}

Let $G=(V, E)$ be a graph, where $V$ is the set of nodes and $E$ is the set of edges. Let $T_{1}=\left(V_{1}, E_{1}\right)$ and $T_{2}=\left(V_{2}, E_{2}\right)$ be two rooted trees and let $u_{1,} v_{1} \in V_{1}$ and $u_{2,} v_{2} \in V_{2}$ be distinct nodes of the respective trees. The tree association graph $(T A G)$ of $T_{1}$ and $T_{2}$ is the graph $G=(V, E)$ where $\mathrm{V}=V_{1} \times V_{2}$, and TAG nodes $\left(u_{1,} u_{2}\right)$ and $\left(v_{1}, v_{2}\right)$ are adjacent when the connectivity between $u_{1}$ and $v_{1}$ is equivalent to that of $u_{2}$ and $v_{2}$. Pelillo et al define equivalence between two sets of nodes in respective trees by comparing path length and level difference in the tree hierarchy. By this definition, there exists one to one correspondence between maximal subtree isomorphism and maximal clique of the TAG from the two trees. Searching for the maximal clique in TAG is equivalent to tree matching.

Next, let $T(V, E, \alpha)$ be an attributed tree, where $\alpha$ is a function which assigns an attribute vector $\alpha(u)$ to each node $u \in V$ Let $\sigma$ be a similarity measure in attribute space. Subtree isomorphism with the largest similarity is called "maximum similarity subtree isomorphism". The weighted TAG (WTAG) of two attributed trees $T_{1}$ and $T_{2}$ is the weighted graph $G=(V, E, \omega)$, where $\omega$ is a function which assigns a positive weight to each node $\mathrm{z}=(u, v) \in V$ as follows:

$$
\omega(z)=\omega(\mathrm{u}, \mathrm{v})=\sigma\left(\alpha_{1}(u), \alpha_{2}(v)\right)
$$

Weight matrix $W=\left(m_{i j}\right)$ is defined as follows:

$$
\begin{array}{ll}
m_{i j}=1-0.5 \sigma_{\min } / \omega\left(u_{\mathrm{i}}\right) & \text { if } i=j, \\
m_{i j}=1 & \text { if } i \neq j \text { and } u_{\mathrm{i}} \text { are adjacent to } u_{\mathrm{j}} \\
0 \leq m_{i j}<1-0.5 \sigma_{\min } /\left(\omega\left(u_{\mathrm{i}}\right)+\omega\left(u_{\mathrm{j}}\right)\right) & \text { otherwise, }
\end{array}
$$

where $\sigma_{\min }$ denotes the minimum value of the similarity measure, $\sigma$.

Pelillo et al. used the following method to search for a maximum clique from weighted TAG. Let $G=(V, E, \omega)$, be an arbitrary weighted graph of order n. The characteristic vector of any subset of nodes $C \subseteq V$, denoted $x^{c}$, is defined as follows:

$$
\begin{aligned}
\mathrm{x}_{i}^{\mathrm{c}} & =\omega\left(u_{\mathrm{i}}\right) / \Omega(C), & & \text { if } u_{\mathrm{i}} \in C \\
& =0, & & \text { otherwise, }
\end{aligned}
$$

where $\Omega(C)$ is the total weight on $\mathrm{C}$. It has been proved that $\mathrm{C}$ is a maximum weight clique of $\mathrm{G}$ if and only if $\boldsymbol{x}^{\mathrm{c}}$ is a global maximizer of the function $x^{T} W x$, where $x^{T} \mathrm{de}-$ notes matrix transposition [7], [8]. Pelillo et al used replicator dynamic system to seek the maximizer [9].

\subsection{Modification of Weighted TAG for Bronchial Nomenclature}

Pelillo et al. defined TAG-node adjacency as an exact agreement between the connectivity of two nodes in one tree and that of their corresponding nodes in the other tree [6]. We propose an alternative definition of TAG-node adjacency to be constructed for the purpose of bronchial nomenclature. Here, a relationship function $r$ between nodes $u, \mathrm{v}$, and $\mathrm{w}$ in a rooted tree is defined as follows:

When $u$ is located upstream from $v$,

$$
\begin{aligned}
& r(u, v)=1, \\
& r(v, u)=-1
\end{aligned}
$$

When $u$ is located neither upstream nor downstream of $w$,

$$
\mathrm{r}(u, w)=\mathrm{r}(w, u)=0 \text {. }
$$


Another relationship function, $q$ in a reference tree is defined as follows:

The basic relationship is the same as $\mathrm{r}$, however, when $u$ and $v$ are segmental nodes,

$$
q(u, v)=q(v, u)=2,
$$

When $u$ is a node having no nomenclature,

$$
q(u, v)=q(v, u)=3
$$

Both of those relationship functions are applicable to multiple branching. From two relationship functions, the adjacency of TAG nodes $A$ is defined as follows:

$$
\begin{array}{ll}
A=1 & \text { if } q<2 \text { and } r=q, \\
A=1 & \text { if } q=2 \text { and } r=0, \\
A=0 & \text { if } q=2 \text { and } r \neq 0, \text { or } q<2 \text { and } r \neq q, \\
A=0 & \text { if } q=3
\end{array}
$$

The definition of the lobar node in the algorithm is slightly different from the anatomical definition of the lobar bronchi. The bilateral lower lobe bronchi are very short, and sometimes the superior segment bronchi of the lower lobe (B6) arise from the right intermediate bronchus and left main bronchus. Therefore, instead of the lower lobe bronchi, the basal bronchi are used in the algorithm. The reference tree is indicated in Figure 4 . There are 30 branches having anatomical names excluding two lower lobe bronchi.

The node attribute vector $\alpha(u)$ of a tree is constructed by the position of a node, denoted $P_{u}$, and the direction of the upward edge, denoted $V_{u}$, The similarity measure $\sigma$ is defined as follows:

$$
\begin{aligned}
& \sigma\left(\alpha\left(u_{1}\right), \alpha\left(u_{2}\right)\right)=1-\beta_{1}\left(1-\left(V_{u}, V_{u 2}\right)\right)-\beta_{2}\left|P_{u 1}-P_{u 2}\right| \\
& \sigma(\mathrm{u}, \mathrm{v})=\sigma_{\min }, \text { if } \sigma(\mathrm{u}, \mathrm{v})<\sigma_{\min },
\end{aligned}
$$

where $\beta_{1}, \beta_{2}$, and $\sigma_{\min }(>0)$ are determined experimentally as $0.5,0.1 / \mathrm{cm}$, and 0.1 , respectively. In order to compare node positions in different trees, size normalization and approximate registration are necessary. The practical methods are described in the next section.

\subsection{Correction of Labeled Node}

In the above algorithm, a descendent of a true segmental node is labeled as the segmental node when its similarity is higher than that of the true segmental node. Therefore, it is necessary to check whether there is a true segmental node in ascendants of a labeled node. Since all descendents of a segmental node are not those of any other segmental nodes, a sibling of a segmental node should have at least one different segmental node in its descendents including itself. Therefore, if the sibling does not, one of its ancestors should be the true segmental node. Thus, correction of a segmental node is performed by replacing the segmental node upwards until a sibling having at least one segmental node is found. If the parent is labeled as a lobar node in spite of the fact its sibling does not have any segmental node, the sibling and its descendents are regarded as belonging to an unknown segmental node. If there are unknown segmental nodes after checking all labeled segmental nodes, a nomenclature which has the highest similarity of the unknown node is labeled.

Proximal branches beyond segmental nodes are relabeled using a relationship between lobes and segments as shown in Figure 1. For example, if a node is located upstream of all unilateral segments, the node is assigned as the main bronchus. When false branches are generated in a proximal branch, the above algorithm does not recognize all parts of the branch. However, by adding this step, all proximal branches are obtained excluding the false branches. 


\section{Experiments}

Nine 3D-CT data sets of the human lungs were used for testing. Scanning occurred with lung volume held near total lung capacity and with subjects lying in the supine posture. The slice thickness was $1.25 \mathrm{~mm}$ with $0.6 \mathrm{~mm}$ spacing, and the pixel size ranged from $0.58 \mathrm{~mm}$ to $0.72 \mathrm{~mm}$. All subjects were studied under an approved University of Iowa IRB protocol. Segmentation and skeletonization of the airway was performed by a method reported by Kiraly et al. [10] and Palagy et al. [11], respectively. More than 190 branches were extracted for each case. In most cases, there were several false branches in the proximal portion of the tree, which could be automatically recognized as false branches. However, in some cases, there were several clusters of numerous false branches in the peripheral lung regions distal to segmental bronchi. These peripheral false branches were due to incorrect segmentation at the periphery, and they could not be automatically recognized as false. Therefore, peripheral false branches were manually recognized and excluded from the evaluation. False branches located at the proximal part were evaluated whether they were labeled correctly "false" or not. The gold standards for the bronchial nomenclatures were given by careful observation of the CT images by one of the authors who was a pulmonologist expert at chest $\mathrm{CT}$ images.

An existing 3D mathematical model of the human airway tree [12] was slightly modified and used as a reference tree. The branching pattern was designed to represent a standard airway tree [3], and bilateral sub-superior segmental bronchi were added as shown in Figure 4. Since the maximum thoracic width of this model is fixed at $30 \mathrm{~cm} \mathrm{[12],} \mathrm{size} \mathrm{normalization} \mathrm{was} \mathrm{performed} \mathrm{according} \mathrm{to} \mathrm{the} \mathrm{maximum} \mathrm{thoracic}$ widths in CT images. The approximate registration was performed by matching the carina point in a normalized object tree to that of the reference tree. Automated detection of the carina point was performed by finding the longest branch located at the center of the thorax. Only branchnodes in an extracted airway tree were subjected to the nomenclature-labeling algorithm, and terminal nodes were labeled later. The reason is that the extracted branches extended peripherally to segmental bronchi.

Table 1 shows the number of extracted branches, labeled branches, and correctly labeled branches for each subject. Almost all branches were accurately labeled except for subject 3. Overall accuracy for nine cases was calculated as $95 \%$.

Table 1. Result of automatic labeling of the bronchial tree extracted from 3D-CT data

\begin{tabular}{|l|l|l|l|l|l|l|l|l|l|l|}
\hline Subject & 1 & 2 & 3 & 4 & 5 & 6 & 7 & 8 & 9 & Total \\
\hline $\begin{array}{l}\text { Extracted } \\
\text { Branches }\end{array}$ & 245 & 197 & 203 & 245 & 192 & 327 & 268 & 195 & 301 & 2,173 \\
\hline $\begin{array}{l}\text { Labeled } \\
\text { branches }\end{array}$ & 245 & 197 & 203 & 244 & 192 & 327 & 266 & 195 & 298 & 2,167 \\
\hline $\begin{array}{l}\text { Correctly } \\
\text { Labeled }\end{array}$ & 245 & 169 & 148 & 244 & 192 & 327 & 266 & 195 & 288 & 2,074 \\
\hline $\begin{array}{c}\text { Accuracy } \\
(\%)\end{array}$ & 100 & 96 & 74 & 100 & 100 & 100 & 99 & 100 & 96 & 95 \\
\hline
\end{tabular}

Figures 5, 6, and 7 show bronchial trees in subjects 1,2 , and 3, respectively. In these figures, segmented bronchial regions and their skeletons are superimposed. Each segmental bronchus and its descendents are distinguished by color. Proximal branches beyond segmental bronchi are colored white. Incorrectly labeled branches 
are colored gray. In Figure 5, even though there were several false branches at proximal bronchi, the nomenclature was successfully performed with an accuracy of 100 $\%$. False branches in the proximal bronchi are correctly labeled as false. The left subsuperior bronchus $\left(\mathrm{B}^{*}\right)$ was correctly labeled.

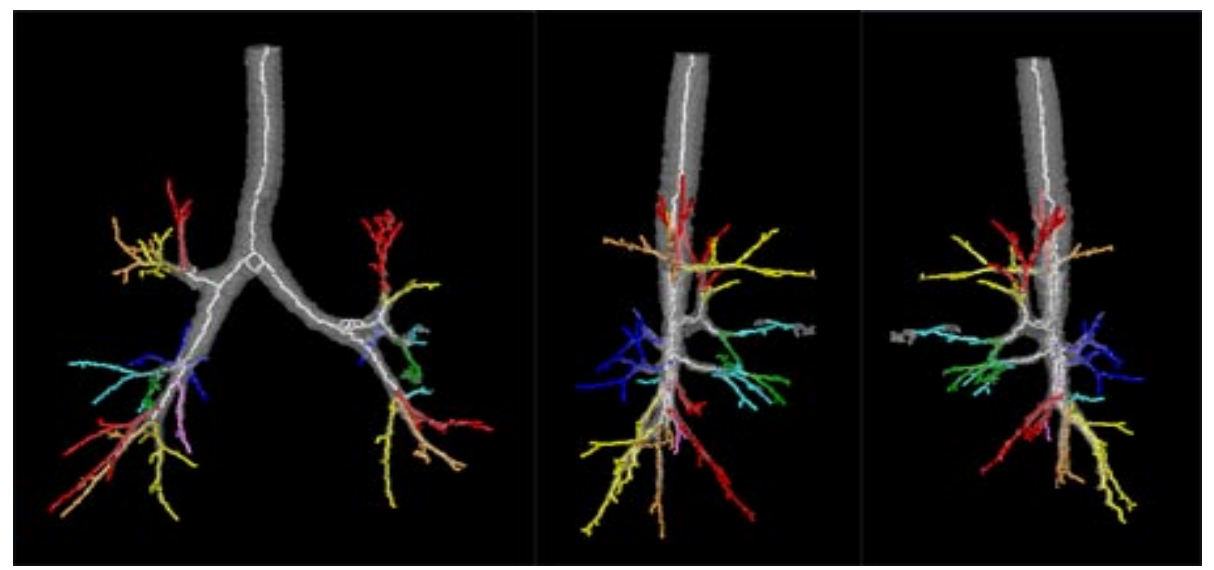

Fig. 5. Labeled bronchial tree in subject 1 . Anterior view (left), right lateral view ( middle), and left lateral view (left). All branches are correctly labeled including false branches

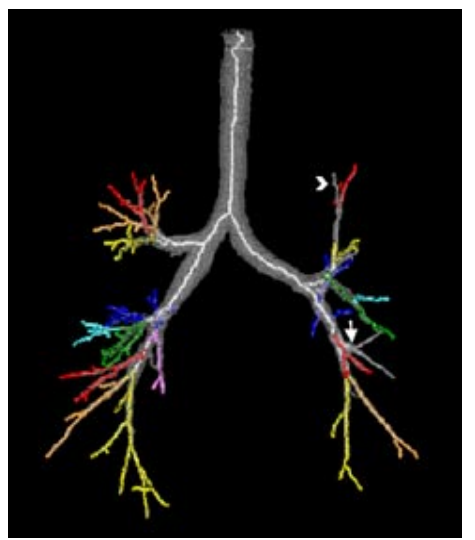

Fig. 6. Labeled bronchial tree in subject 2 .

There are two mislabeled sub-segmental bronchi

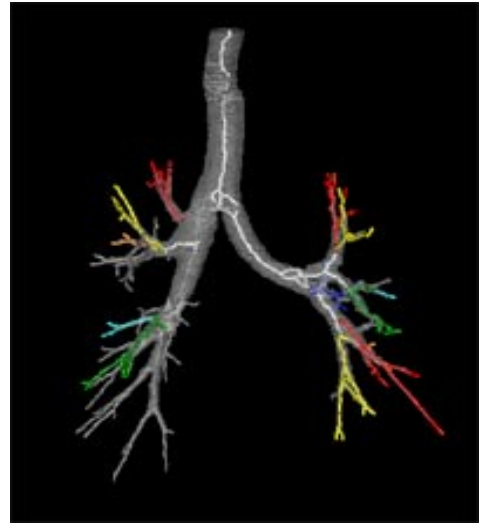

Fig. 7. Labeled bronchial tree in subject 3. The right main and intermediate bronchi are unlabeled. Half of right segmental bronchi are mislabeled

There were several incorrectly labeled branches in subject 2. Mislabeling occurred at the level of the sub-segmental bronchus as shown in Figure 6. Two sub-segmental bronchi of the anterior segmental bronchi of the left lower lobe (B8) arose without having a common trunk in this case. One sub-segmental bronchi were correctly labeled as B8, but the other was labeled as the lateral segmental bronchi of the left lower lobe (B9) as shown by an arrow in Figure 6. One of sub-segmental bronchus of the apico-posterior segmental bronchus of the left upper lobe $(\mathrm{B} 1+2)$ were mislabeled 
as the anterior segmental bronchus of the left upper lobe (B3), because the true B3 arose at the lower position than usual, the apical sub-segmental bronchi of B1+2 was first labeled as B3 as shown by an arrow head. Causes of mislabeling in subjects 7 and 9 are due to lack of a common trunk of sub-segmental bronchi as left B8 in subject 2 .

Subject 3 had a very rare variant branching pattern where the apical segmental bronchi of the right upper lobe (B1) arose from the right main bronchus as shown in Figure 7. Since the right upper lobe was much larger than usual, positions and directions of other branches were different from those in a usual airway tree. Therefore, only half of the branches in the right lung were correctly labeled. The right main bronchus and the intermediate bronchus were unlabeled, as colored gray in Figure 7, because of inconsistency of the relationship between lobe and segments. This indicated that the branching abnormality occurred at the level of the main bronchus. There were two unlabeled branches in the left lung. The reason is that they were terminal branches in the extracted tree although they were sub-segmental bronchi.

\section{Discussion}

The experimental results indicate that the proposed algorithm is useful for bronchial nomenclature labeling up to the segmental level of the airway tree in human CT images with $95 \%$ accuracy.

The lowest accuracy is seen in subject 3, where a very rare branching pattern was observed. According to Yamashita [3], such a pattern did not occur in 170 specimens studied. It is unlikely that automated methods for labeling nomenclature will be successful in such cases, and manual correction by an expert will be required. The proposed algorithm can alert the user when such unusual patterns are encountered by labeling no nomenclatures to proximal bronchi.

Except subject 3, the accuracy of nomenclature was $98 \%$ in average, which is considered satisfactory for practical application. The main cause of mislabeling was the lack of a common trunk of sub-segmental bronchi. Although the mislabeled bronchi in the left lower lobe in subject 2 was recognized as a sub-segmental bronchus of B8 by one of the authors, other experts may name it a sub-segmental bronchi of B9 or $\mathrm{B}^{*}$. Branching patterns of bronchi at the sub-segmental level are more varied than at the segmental level [3], and hence the difficulty of labeling of sub-segmental bronchi is much higher. In order to solve this problem, the extension of the proposed algorithm to the level of sub-segmental level will be useful.

There are three parameters in the weighted TAG that determine the similarity between the reference tree node and an object tree node. Although fixed values were used in the experiment, optimal values should be investigated as the number of clinical cases increase. Accuracy of the nomenclature labeling is also influenced by generality of a reference tree. We used a model-derived tree [12] as a reference in the experiment. The model-derived tree consists of the most common branching pattern in respective lobes and contains accessory segmental bronchi, which will rarely be found in real cases. Refinement of the reference tree is expected by statistically analyzing morphometric data of the airway trees in 3D CT images as we continue to study additional subjects.

Mori et al. proposed a knowledge-based labeling method of the airway tree. Nomenclature labeling in their method is executed in the direction of the periphery from 
the trachea with the depth first search [1]. However, as they discussed in their paper, the depth first search propagates proximal mislabeling into the periphery. Searching for a global solution, as in our algorithm, may be more suitable for bronchial nomenclature labeling. Krass et al. reported that they performed automated bronchial labeling based on graph theory[13], but details of the algorithm was not described in their paper.

Automated nomenclature labeling of the airway tree in 3D-CT images is a promising technique for both clinical and fundamental imaging investigation. One can easily begin to recognize branching patterns and to catalogue the spatial distribution of the airway tree. Although it is difficult to obtain precise segmentation of peripheral small airways, it is possible to label pulmonary arteries adjacent to labeled airways and to track more peripheral branches of pulmonary arteries. Arterial labels can likely be transferred to their adjacent airways. These processes will provide better understanding of segmental anatomy of the lung.

\section{Conclusion}

We have proposed a bronchial nomenclature labeling algorithm that is robust to various branching patterns and false branches that arise during image segmentation and skeletonization. The results show very accurate labeling for more than 200 branches. This technique will be useful for both of clinical and fundamental imaging investigations of the lung.

\section{Acknowledgements:}

This works was supported in part by NIH HL-04368 and HL-060158 and NSF 0092758.

\section{References}

1. Mori, K., Hasegawa, J., Suenaga, Y., Toriwaki J.: Automated Anatomical Labeling of the Bronchial Branch and Its Application to the Virtual Bronchoscopy System. IEEE Trans. Med. Imag. 19 (2000) 103-114

2. Weibel, E.R.: Morphometry of the Human Lung. Academic Press, New York (1963).

3. Yamashita, H.: Roentgenologic Anatomy of the Lung. Igaku-shoin, Tokyo (1978).

4. Moore, K.L. Clinically Oriented Anatomy. Williams \&Willkins, Baltimore (1985) 49-148

5. Agur, A.M.R., Lee, M.J. Grant Atlas of Anatomy, Williams \&Willkins, Baltimore (1991) 1-76.

6. Pelillo, M., Siddiqi, K., Zucker, S.W.: Matching Hierachical Structure Using Association Graph. IEEE Trans. PAMI. 21 (1999) 1105-1120

7. Motzkin, T.S., Straus, E.G.: Maxima for Graphs and a New Proof of a Theorem of Turan. Canadian J. Math. 17 (1965) 533-540

8. Bomze, L.M., Budinich, M., Pardalos, P.M., Pelillo, M.: The Maximum Clique Problem. In: Du, D.-Z., Paradolas, P.M. (eds): Handbook of Combinatorial Optimization, Vol.4. Mass. Kluwer Academic, Boston (1999)

9. Pelillo, M.: The Dynamics of Nonlinear Relaxation Labeling Process. J. Math.Imag. and Vision 7(1997) 309-323

10. 10. Kiraly, A, Higgins, W.E, Hoffman, E.A., McLennan G., Reinhardt G.M.: 3D Human Airway Segmentation for Virtual Bronchoscopy. In Proc. of SPIE Conf on Medical Imaging (2002) (in press) 
11. Palagyi, K., Sorantin, E., Balogh, E., Kuba, A., Halmai, C., Erdohelyi, B., Hausegger, K.:A sequential 3D Thinning Algorithm and its Medical Applications. In 17th Int. Conf. IPMI (2001) 409-415

12. Kitaoka, H., Takaki, R., Suki, B.: A Three-Dimensional Model of the Human Airway Tree. J. Appl Physiol. 87(1999) 2207-2217

13. Krass, S., Selle, D., Boehm D., Jend H.H., Kriete A., Rau W.S., Peitgen H.O.: Determination of Bronchopulmonary Segments Based on HRCT Data. In: Lemke H.U. et al (eds): Computer Assisted Radiology and Surgery. Elsevier, Amsterdam (2000) 584-589. 